\title{
A CASE OF CORONVIRUS DISEASE 2019 WITH PSYCHOLOGICAL DISORDERS
}

\author{
Jiaxiang Chen ${ }^{1, *}$, Chong Tian ${ }^{2, *}$, Xinge Cheng ${ }^{3}$, Ying Huang ${ }^{4}$, Lei Tang ${ }^{2}$, \\ Rongpin Wang ${ }^{2} \&$ Xianchun Zeng ${ }^{2,5}$ \\ ${ }^{1}$ Medical College, Guizhou University, Guiyang, China \\ ${ }^{2}$ Department of Radiology, Guizhou Provincial People's Hospital, Guiyang, China \\ ${ }^{3}$ Department of Graduate School, Zunyi Medical University, Zunyi, China \\ ${ }^{4}$ Department of Psychology, Guizhou Provincial People's Hospital, Guiyang, China \\ ${ }^{5}$ Guizhou Jiangjunshan Hospital, Guiyang, China
}

*Jiaxiang Chen and Chong Tian contributed equally to this work

received: 17.8.2020;

revised: 18.9.2020;

accepted: 20.10 .2020

\section{INTRODUCTION}

In December 2019, an outbreak of Coronvirus Disease 2019 (COVID-19) caused by SARS-CoV-2 emerged in Wuhan, China. Human-to-human transmission through droplets and personal contacts quickly caused more than 18 million infections in 8 months thereafter ( $\mathrm{Zu}$ et al. 2020), and the number was still increasing exponentially. During the outbreak, more and more patients with COVID-19 began to develop psychological disorders. As a response, the National Health Commission of China issued several guidelines for psychological crisis interventions (National Health Commission of China 2020a,b). However, the psychological issues often received less attention compared with the physiological impact of pneumonia during the epidemic. Here, we report a case of COVID-19 with psychological disorders.

\section{CASE DESCRIPTION}

A 49-year-old female was admitted to Liupanshui People's Hospital with fever and pharyngeal discomfort on January 22, 2020. The patient underwent a duodenal resection in Wuhan 20 days ago for pancreatic serous cystadenoma, and had a medical history of bowel repair and cholecystectomy. She had a body temperature of $37.5^{\circ}$, and laboratory tests showed normal leukocyte counts $\left(4.18 \times 10^{9} / \mathrm{L}\right)$, decreased lymphocyte counts $\left(0.51 \times 10^{9} / \mathrm{L}\right)$, and elevated $\mathrm{C}$-reactive protein (43.7 mg/L). Chest computed tomography (CT) showed signs of lung infection. She was finally diagnosed with COVID-19 based on the real-time reverse transcription polymerase chain reaction (RT-PCR) results. After treatment with Lopinavir/Ritonavir combined with interferon inhalation, her body temperature returned normal.

On February 5, the patient was transferred to Jiangjunshan Hospital (A designated hospital for COVID-19 treatment in Guizhou Province). Chest CT showed highdensity patchy shadows in the posterior basal segment of the lower lobe of the right lung (Figure 1A), and the treat- ment was continued as above. On day 3 of hospitalization, the patient began to suffer from anxiety, depression and insomnia, which became worsened after knowing the positive RT-PCR result. The psychological symptoms were relieved by psychotherapy combined with oral estazolam and caloric supplementation. In subsequent treatment, however, these symptoms recur red. On day 9 of hospitalization, two attending psychiatrists conducted a clinical interview. The patient reported feeling guilty, worries about her health and often wailed in the ward. During the interview, the patients displayed anxiety. According to The Diagnostic and Statistical Manual of Mental Disorders, Fifth Edition (DSM-5), the patient was diagnosed with major depressive disorder. On day 11 of hospitalization, follow-up CT examinations showed substantial absorption of pulmonary lesions (Figure 1B) and postoperative changes of the duodenal pancreas (Figure 1C, 1D). After psychotherapy combined with anti-depression therapy (oral escitalopram oxalate) and sedation (oral lorazepam), these psychological symptoms gradually improved and remitted. On day 17 of hospitalization (February 22), two pharyngeal swab RT-PCR tests (interval $>24 \mathrm{~h}$ ) both had negative results. Thus, the patient was discharged.

This report was approved by Guizhou Jiangjunshan Hospital Institutional Review Board and conforms to the provisions of the Declaration of Helsinki. The requirement for informed consent was waived.

\section{DISCUSSION}

The response of the general public to infectious diseases, especially those with extremely-high morbidity and mortality, is the distancing-blame-stigma pattern, including distancing oneself from the diseases, blaming persons for disease's origin and spread, and stigmatization of patients (Joffe 2011). They, combined with the patients' concerns about the health, may cause psychological disorders in patients, including feelings of guilt and shame, and symptoms of anxiety, depression, and insomnia (Hawryluck 2004, Ji 2017). 


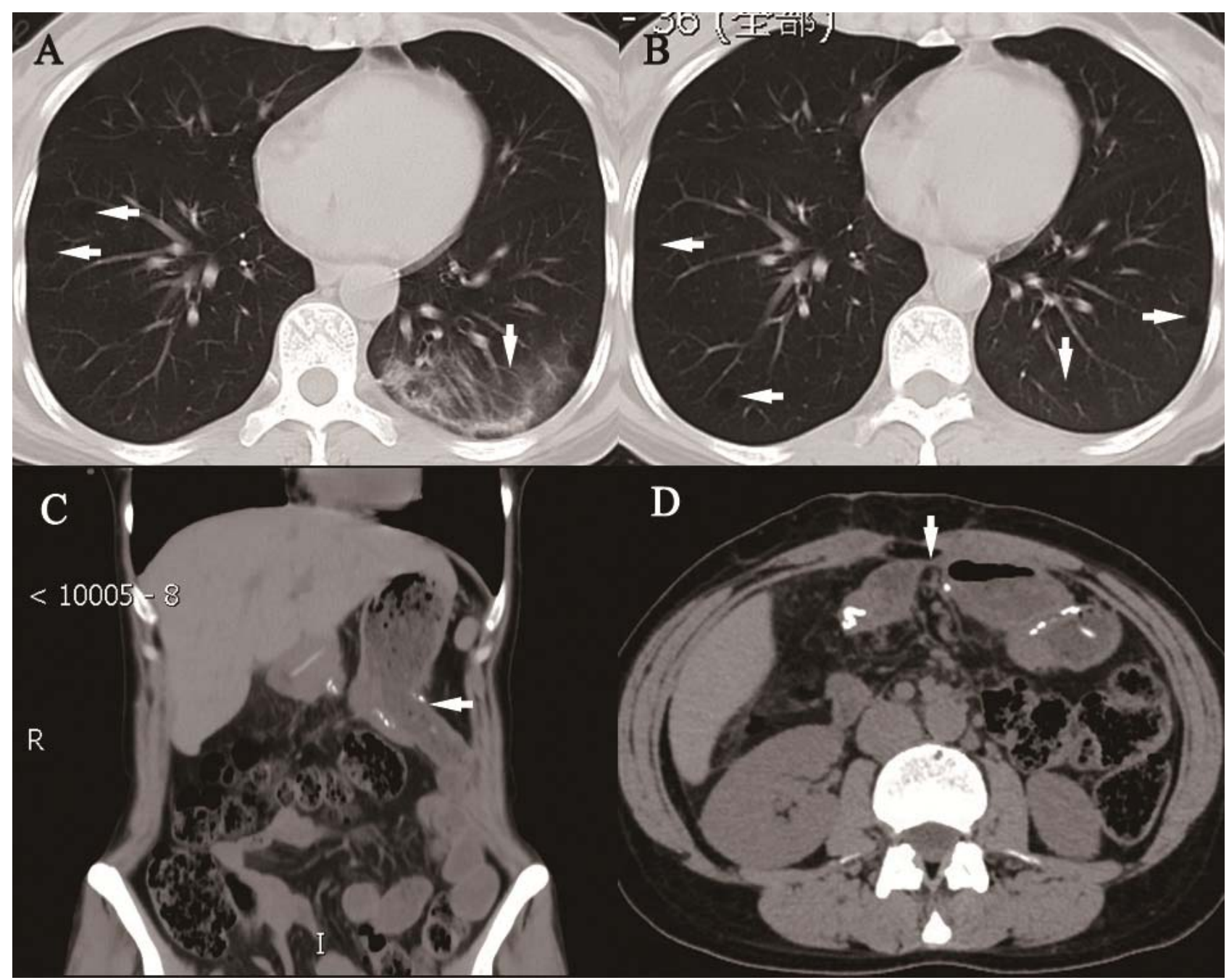

Figure 1. Chest computed tomography (CT) images of the 49-year-old patient with Coronvirus Disease 2019 (COVID-19) (A) Initial CT showed high-density patchy shadows in the posterior basal segment of the lower lobe of the right lung (vertical arrows) and scattered low-density cystic shadows in bilateral lungs (horizontal arrow). (B) Follow-up chest CT (on the $11^{\text {th }}$ day of hospitalization) showed substantial absorption of pulmonary lesions (vertical arrow) and scattered low-density cystic shadows in bilateral lungs (horizontal arrows). (C. D) Postoperative changes of the duodenal pancreas (arrow)

Patients with psychological disorders may develop new social, behavioral, and psychological issues, including the posttraumatic stress disorder, and distrust of other individuals for disease spread and of the healthcare services for their abilities to contain the outbreak (Ho 2020). Psychological disorders can also affect the disease course and prognosis. Incorrect cognitive perceptions may lead to decreased treatment adherence in patients, and negative emotions may lead to worsening of pain, affecting the patient self-reported outcomes (Sawyer 2011, Surdea-Blaga 2016). Moreover, the increased stress hormones due to chronic psychological stress may cause dysregulations of cytokines, leading to immunosuppression, slower wound healing and antiviral deficits. Chronic stress can also lead to inflammation and exacerbation of disease through increased proinflammatory cytokines (Schneiderman 2005).

The psychological crisis interventions for patients with COVID-19 include stabilizing emotions, guiding correct cognitive perceptions of disease and treatment, and building self-confidence (National Health Commission of China 2020 b). High self-efficacy (confidence in treatment), correct cognitive perception and accurate outcome expectations can promote treatment adherence (Sawyer 2011). In this report, the factors affecting the psychological disorders of the patient may be the severity of physical disease and psychotherapy. The anxiety, depression and insomnia symptoms of the patient may arise from the physical duodenal resection and the slow progression of COVID-19. After psychological interventions and drug therapy, the psychological disorders improved.

In conclusion, here we reported a case of COVID-19 with major depressive disorder. The patient exhibited anxiety, depression and insomnia. After psychological interventions and drug therapy, the psychological disorders improved and remitted. The factors affecting the psychological disorders of patient with COVID-19 may 
be the severity of physical disease and the administration of psychotherapy. We therefore recommend the initiation of psychological interventions in COVID-19 patients with psychological disorders, in the aim to establish excellent self-efficacy, correct cognition perception and accurate outcome expectation, which will have a positive effect on psychological health and disease treatment.

\section{Acknowledgements:}

This study was supported by the Guizhou Science and Technology Project (QKHZC[2020]4Y002), and the Guiyang Science and Technology Project (ZKXM[2020]4).

Conflict of interest: None to declare.

\section{Contribution of individual authors:}

Writing-original draft: Jiaxiang Chen \& Chong Tian. Writing-review and editing: Xinge Cheng \& Ying Huang.

Investigation: Lei Tang, Rongpin Wang \& Xianchun Zeng.

Supervision: Xianchun Zeng.

\section{References}

1. Guidelines for psychological assistance hotlines during 2019-nCoV pneumonia epidemic. http://www.nhc.gov.cn/ $\mathrm{jkj} / \mathrm{s} 3577 / 202002 / f 389 f 20 c c 1174 b 21 \mathrm{~b} 981$ ea2919beb8b0.s html. Updated 2020 a. Accessed July 1, 2020
2. Hawryluck L, Gold WL, Robinson S, Pogorski S, Galea S \& Styra R: SARS control and psychological effects of quarantine, Toronto, Canada. Emerg Infect Dis 2004; 10:1206-12

3. Ho CS, Chee CY \& Ho RC: Mental Health Strategies to Combat the Psychological Impact of COVID-19 Beyond Paranoia and Panic. Ann Acad Med Singapore 2020; 49:1-3

4. Ji D, Ji YJ, Duan XZ, Li WG, Sun ZQ, Song XA et al: Prevalence of psychological symptoms among Ebola survivors and healthcare workers during the 2014-2015 Ebola outbreak in Sierra Leone: a cross-sectional study. Oncotarget 2017; 8:12784-91

5. Joffe H: Public apprehension of emerging infectious diseases: are changes afoot? Public Underst Sci 2011; 20:446-460

6. Sawyer AM, Gooneratne NS, Marcus CL, Ofer D, Richards KC \& Weaver TE: A systematic review of CPAP adherence across age groups: clinical and empiric insights for developing CPAP adherence interventions. Sleep Med Rev 2011; 15:343-56

7. Schneiderman N, Ironson $G$ \& Siegel SD: Stress and health: psychological, behavioral, and biological determinants. Annu Rev Clin Psyc 2005; 1:607-28

8. Surdea-Blaga T, Baban A, Nedelcu L \& Dumitrascu DL: Psychological Interventions for Irritable Bowel Syndrome. $J$ Gastrointestin Liver Dis 2016; 25:359-66

9. The guidelines of psychological crisis intervention for COVID-19 pneumonia. http://www.nhc.gov.cn/jkj/ s3577/202001/6adc08b966594253b2b791be5c3b9467.sht ml. Updated 2020b. Accessed July 1, 2020

10. Zu ZY, Jiang MD, Xu PP, Chen W, Ni QQ, Lu GM et al: Coronavirus Disease 2019 (COVID-19): A Perspective from China [published online ahead of print]. Radiology 2020; 200490

Correspondence:

Xianchun Zeng, MD

Department of Radiology, Guizhou Provincial People's Hospital

No.83, Zhongshandong Road, Guiyang, 550002, China

E-mail: zengxianchun04@foxmail.com 